\title{
Approximation of functions by singular integrals
}

\author{
Rahim M. Rzaev ${ }^{1,2}$, Gulnara Kh. Mammadova ${ }^{3}$, Mansur Sh. Maharramov ${ }^{1}$ \\ ${ }^{1}$ Institute of Mathematics and Mechanics of National Academy of Sciences of Azerbaijan, Baku, AZ1141, Azerbaijan \\ ${ }^{2}$ Department of Mathematics and Informatics of Azerbaijan State Pedagogical University, Baku, AZ1000, Azerbaijan \\ ${ }^{3}$ Department of Mathematics and Informatics of Ganja State University, Ganja, Azerbaijan
}

Email address:

rrzaev@rambler.ru (R. M. Rzaev)

To cite this article:

Rahim M. Rzaev, Gulnara Kh. Mammadova, Mansur Sh. Maharramov. Approximation of Functions by Singular Integrals. Pure and Applied Mathematics Journal .Vol. 3, No. 6, 2014, pp. 113-120. doi: 10.11648/j.pamj.20140306.11

Abstract: In this work questions on approximation of locally summable functions by singular integrals are investigated. Was estimated the rate of approximation in terms of various metric characteristics describing the structural properties of the given function.

Keywords: Approximation, Singular Integrals, Bounded Mean Oscillation, Vanishing Mean Oscillation

\section{Introduction}

Let $L\left(R^{n}\right)$ be class of function which is integrable on Euclidean space $R^{n}$. The function $K \in L\left(R^{n}\right)$ is called kernel if

$$
\int_{R^{n}} K(x) d x=1
$$

Let $x \in R^{n}, \varepsilon>0, K_{\varepsilon}(x)=\varepsilon^{-n} K\left(\frac{x}{\varepsilon}\right)$ and $K_{\varepsilon} f(x)=$

$$
\left(K_{\varepsilon} * f\right)(x)=\int_{R^{n}} K_{\varepsilon}(x-t) f(t) d t \text {, }
$$

where $f$ is locally integrable function such that for all $\varepsilon>0$ the integral on the right-hand side is finite.

In this work questions on approximation of locally summable functions by singular integrals of type (1.1) are investigated. Was estimated the rate of approximation in terms of various metric characteristics describing the structural properties of the given function.

Note that various aspects of questions on approximation of function $f$ by singular integrals of a kind (1.1) have been investigated in works of many authors (see, e.g., [1], [2], [3], [5], [8], [9], [10], [15], [16] and the literature quoted there).

\section{Some Definitions, Notation and Preliminary Facts}

Let $f$ be a locally integrable on $R^{n}$ function, i.e. $f \in L_{l o c}\left(R^{n}\right), B(x, r)$ closed ball in $R^{n}$ with center $x \in R^{n}$ and radius $r>0$, i.e.

$$
\begin{aligned}
B(x, r) & :=\left\{y \in R^{n}:|x-y| \leq r\right\}, \\
f_{B(x, r)} & =\frac{1}{|B(x, r)|} \int_{B(x, r)} f(t) d t \\
\Omega(f, B(x, r)) & =\frac{1}{|B(x, r)|} \int_{B(x, r)}\left|f(t)-f_{B(x, r)}\right| d t,
\end{aligned}
$$

where $|B(x, r)|$ denotes the volume of a ball $B(x, r)$.

For $f \in L_{l o c}\left(R^{n}\right)$ and $x_{0} \in R^{n}$ introduce the following notation ([10], [11]):

$$
m_{f}\left(x_{0} ; \delta\right)=\sup \{\Omega(f, B(x, r)): \quad r \leq \delta\}(\delta>0) .
$$

It is obvious that, $m_{f}\left(x_{0} ; \delta\right)$ is monotone increasing on interval $(0,+\infty)$ due to the argument $\delta$.

The point $x_{0} \in R^{n}$ is called $d$-point of $f \in L_{l o c}\left(R^{n}\right)$, if there exists a finite $\operatorname{limit}_{\lim _{r \rightarrow 0}} f_{B\left(x_{0}, r\right)}=s_{f}\left(x_{0}\right)$. The collection of all $d$-points of $f$ is denoted by $D(f)$.

The point $x_{0} \in R^{n}$ is called Lebesgue point (or l-point) of function $f \in L_{l o c}\left(R^{n}\right)$, if there exists a number $l_{f}\left(x_{0}\right)$, such that

$$
\lim _{r \rightarrow 0} \frac{1}{\left|B\left(x_{0}, r\right)\right|} \int_{B\left(x_{0}, r\right)}\left|f(t)-l_{f}\left(x_{0}\right)\right| d t=0 .
$$

Set of all $l$-points of function $f$ is denoted by $L(f)$. It is 
clear that if $x_{0} \in L(f)$, then $x_{0} \in D(f)$ and $s_{f}\left(x_{0}\right)=$ $l_{f}\left(x_{0}\right)$.

Point $x_{0} \in R^{n}$ we will name a $m$-point of the function $f \in L_{l o c}\left(R^{n}\right)$ (see [10]), if $\lim _{r \rightarrow 0} m_{f}\left(x_{0} ; r\right)=0$. Set of all $m$-points of function $f \in L_{l o c}\left(R^{n}\right)$ is denoted by $M(f)$.

Theorem A [10], [12]. If $f \in L_{l o c}\left(R^{n}\right)$, then the following equality is satisfied:

$$
L(f)=D(f) \cap M(f) .
$$

For $f \in L_{l o c}\left(R^{n}\right)$ and $x_{0} \in D(f)$ we also introduce the following notation

$$
\begin{gathered}
\omega_{f}\left(x_{0} ; \delta\right)=\sup _{0<r \leq \delta} \frac{1}{\left|B\left(x_{0}, r\right)\right|} \int_{B\left(x_{0}, r\right)}\left|f(t)-s_{f}\left(x_{0}\right)\right| d t, \\
\delta>0 .
\end{gathered}
$$

It easy to see that $\omega_{f}\left(x_{0} ; \delta\right)$ is monotone increasing on interval $(0,+\infty)$ due to the argument $\delta$. It is also easy to see that the point $x_{0} \in R^{n}$ is $l$-point of a function $f$ if and only if $\lim _{r \rightarrow 0} \omega_{f}\left(x_{0} ; r\right)=0$.

Now let's note some facts which we will use in the future.

Theorem B [10]. Let $K \in L\left(R^{n}\right)$ be kernel and

$$
\begin{gathered}
k(x)=\operatorname{esssup}\{|K(y)|: \quad|y| \geq|x|\}, k \in L\left(R^{n}\right), k_{0}(|x|)= \\
k(x),
\end{gathered}
$$

$f \in L_{l o c}\left(R^{n}\right), \quad x_{0} \in R^{n}$. If right hand side integrals are convergent, then the following inequality holds:

$$
\begin{gathered}
\left|K_{\varepsilon} f\left(x_{0}\right)-f_{B\left(x_{0}, \varepsilon\right)}\right| \leq \\
\leq c\left(n, k_{0}\right)\left(m_{f}\left(x_{0} ; \varepsilon\right)+\int_{0}^{\infty} x^{n-1} k_{0}(x) m_{f}\left(x_{0} ; 4 \varepsilon x\right) d x+\right. \\
+\int_{0}^{\varepsilon} \frac{m_{f}\left(x_{0} ; t\right)}{t}\left(\int_{0}^{\frac{t}{4 \varepsilon}} x^{n-1} k_{0}(x) d x\right) d t+ \\
\left.+\int_{\varepsilon}^{\infty} \frac{m_{f}\left(x_{0} ; t\right)}{t}\left(\int_{\frac{t}{4 \varepsilon}}^{\infty} x^{n-1} k_{0}(x) d x\right) d t\right), \varepsilon>0
\end{gathered}
$$

where $c\left(n, k_{0}\right)$ is constant depending only on the function $k_{0}$ and dimension $n$.

Note that the function

$$
P(x)=\pi^{-(n+1)} \Gamma\left(\frac{n+1}{2}\right)\left(|x|^{2}+1\right)^{-\frac{n+1}{2}}, \quad x \in R^{n},
$$

is called the Poisson kernel. If $K(x) \equiv P(x), x \in R^{n}$, then it is obvious, that $k(x) \equiv P(x), \quad x \in R^{n}$, and

$$
k_{0}(t)=\pi^{-(n+1)} \Gamma\left(\frac{n+1}{2}\right)\left(t^{2}+1\right)^{-\frac{n+1}{2}}, t \in[0,+\infty) .
$$

Let $f \in L_{l o c}\left(R^{n}\right)$ and

$$
M_{f}(\delta):=\sup \left\{m_{f}(x ; \delta): x \in R^{n}\right\}, \delta>0 .
$$

A function $M_{f}(\delta)$ is called modulus of mean oscillation of function $f$. Note that the function $M_{f}(\delta)$ was firstly introduced in [14]. If $\varphi(\delta)$ is positive function which is monotone increasing on interval $(0,+\infty)$, then $B M O_{\varphi}=$ $B M O_{\varphi}\left(R^{n}\right)$ denotes the set of all functions $f \in L_{l o c}\left(R^{n}\right)$, for which the condition (see, for example, [6])

$$
\|f\|_{B M O_{\varphi}}:=\sup \left\{\frac{M_{f}(\delta)}{\varphi(\delta)}: \quad \delta>0\right\}<+\infty
$$

satisfied.

If we consider the class $B M O_{\varphi}$ as subset in the quotient space $L_{\text {loc }}\left(R^{n}\right) /$ constants\}, then $\|\cdot\|_{B M O_{\varphi}}$ is the norm in $B M O_{\varphi}$ and in this norm $B M O_{\varphi}$ is Banach space. If $\varphi(\delta) \equiv 1$, then $B M O_{\varphi}$ turns to space $B M O$, which was firstly introduced in [7].

We also introduce the class [13]

$$
V M O=\operatorname{VMO}\left(R^{n}\right):=\left\{f \in B M O: \lim _{\delta \rightarrow+0} M_{f}(\delta)=0\right\}
$$

with norm $\|f\|_{V M O}:=\|f\|_{B M O}$.

\section{On Approximation in Terms of the Characteristics $\omega_{f}\left(x_{0} ; \delta\right)$}

Theorem 3.1. Let $K \in L\left(R^{n}\right)$ be kernel,

$$
\begin{gathered}
k(x)=\operatorname{esssup}\{|K(y)|:|y| \geq|x|\}, \quad k \in L\left(R^{n}\right), \\
k_{0}(|x|)=k(x),
\end{gathered}
$$

$f \in L_{l o c}\left(R^{n}\right), x_{0} \in R^{n}$ is $l$-point of function $f$. If right hand side integrals are convergent, then the following inequality holds:

$$
\begin{gathered}
\left|K_{\varepsilon} f\left(x_{0}\right)-s_{f}\left(x_{0}\right)\right| \leq \\
\leq c \cdot \varepsilon^{-n} \int_{0}^{\infty} t^{n-1} k_{0}\left(\frac{t}{\varepsilon}\right) \omega_{f}\left(x_{0} ; 4 t\right) d t, \quad \varepsilon>0,
\end{gathered}
$$

where $\mathrm{c}$ is a positive constant depending only on the dimension $n$.

Proof. We have

$$
\begin{gathered}
\left|K_{\varepsilon} f\left(x_{0}\right)-s_{f}\left(x_{0}\right)\right|= \\
=\left|\int_{R^{n}} K_{\varepsilon}\left(x_{0}-t\right)\left[f(t)-s_{f}\left(x_{0}\right)\right] d t\right| \leq \\
\leq \varepsilon^{-n} \int_{R^{n}}\left|K\left(\frac{x_{0}-t}{\varepsilon}\right)\right|\left|f(t)-s_{f}\left(x_{0}\right)\right| d t \leq \\
\leq \varepsilon^{-n} \int_{R^{n}} k_{0}\left(\left|\frac{x_{0}-t}{\varepsilon}\right|\right)\left|f(t)-s_{f}\left(x_{0}\right)\right| d t= \\
=\sum_{m=-\infty}^{\infty} \varepsilon^{-n} \int_{2^{m}} k_{\varepsilon<\left|x_{0}-t\right| \leq 2^{m+1} \varepsilon} k_{0}\left(\left|\frac{x_{0}-t}{\varepsilon}\right|\right) \mid f(t)- \\
-s_{f}\left(x_{0}\right) \mid d t=: \sum_{m=-\infty}^{\infty} \tau_{m} .
\end{gathered}
$$


We estimate each of the terms $\tau_{m}, m=0, \pm 1, \pm 2, \ldots$. Considering that, $k_{0}(t)$ monotone decreasing function on interval $(0,+\infty)$, we get

$$
\begin{gathered}
\tau_{m}=\varepsilon^{-n} \int_{2^{m}{ }_{\varepsilon<\left|x_{0}-t\right| \leq 2^{m+1} \varepsilon}} k_{0}\left(\frac{\left|x_{0}-t\right|}{\varepsilon}\right)\left|f(t)-s_{f}\left(x_{0}\right)\right| d t \leq \\
\leq k_{0}\left(2^{m}\right) \cdot \frac{1}{\varepsilon^{n}} \int_{B\left(x_{0}, 2^{m+1} \varepsilon\right)}\left|f(t)-s_{f}\left(x_{0}\right)\right| d t= \\
=k_{0}\left(2^{m}\right) \cdot \frac{\left(2^{m+1}\right)^{n} \cdot|B(0,1)|}{|B(0,1)| \cdot\left(2^{m+1} \varepsilon\right)^{n}} \times \\
\quad \times \quad \int_{B\left(x_{0}, 2^{m+1} \varepsilon\right)}\left|f(t)-s_{f}\left(x_{0}\right)\right| d t \leq \\
=k_{0}\left(2^{m}\right) \cdot\left(2^{m+1}\right)^{n} \cdot|B(0,1)| \cdot \omega_{f}\left(x_{0} ; 2^{m+1} \varepsilon\right) .
\end{gathered}
$$

Thus, by (3.2)

$$
\begin{gathered}
\left|K_{\varepsilon} f\left(x_{0}\right)-s_{f}\left(x_{0}\right)\right| \leq|B(0,1)| \times \\
\times \sum_{m=-\infty}^{\infty}\left(2^{m+1}\right)^{n} \cdot k_{0}\left(2^{m}\right) \cdot \omega_{f}\left(x_{0} ; 2^{m+1} \varepsilon\right) .
\end{gathered}
$$

On the other hand, we have

$$
\begin{gathered}
\varepsilon^{-n} \int_{0}^{\infty} t^{n-1} k_{0}\left(\frac{t}{\varepsilon}\right) \omega_{f}\left(x_{0} ; 4 t\right) d t= \\
=\sum_{m=-\infty}^{\infty} \varepsilon^{-n} \int_{2^{m-1} \varepsilon}^{2^{m} \varepsilon} t^{n-1} k_{0}\left(\frac{t}{\varepsilon}\right) \omega_{f}\left(x_{0} ; 4 t\right) d t \geq \\
\geq \sum_{m=-\infty}^{\infty} \varepsilon^{-n} \cdot k_{0}\left(2^{m}\right) \omega_{f}\left(x_{0} ; 4 \cdot 2^{m-1} \varepsilon\right) \int_{2^{m-1} \varepsilon}^{2^{m} \varepsilon} t^{n-1} d t= \\
=\frac{2^{n}-1}{n \cdot 4^{n}} \sum_{m=-\infty}^{\infty} k_{0}\left(2^{m}\right)\left(2^{m+1}\right)^{n} \omega_{f}\left(x_{0} ; 2^{m+1} \varepsilon\right) .
\end{gathered}
$$

Combining inequalities (3.3) and (3.4) we get inequality (3.1) with constant $c=|B(0,1)| \cdot \frac{n \cdot 4^{n}}{2^{n}-1}$.

Corollary 3.1. Let kernel $K(x)$ satisfies the conditions of Theorem 3.1, $f \in L_{l o c}\left(R^{n}\right), x_{0} \in R^{n}$ is Lebesgue point of function $f$ and

$$
\int_{1}^{\infty} k_{0}(t) t^{n-1} \omega_{f}\left(x_{0} ; 4 t\right) d t<+\infty
$$

Then $\lim _{\varepsilon \rightarrow 0} K_{\varepsilon} f\left(x_{0}\right)=l_{f}\left(x_{0}\right)$.

Proof. Let $0<\varepsilon \leq 1$. Then we have

$$
\begin{gathered}
\varepsilon^{-n} \int_{0}^{\infty} t^{n-1} k_{0}\left(\frac{t}{\varepsilon}\right) \omega_{f}\left(x_{0} ; 4 t\right) d t= \\
=\varepsilon^{-n} \int_{0}^{\infty} \varepsilon^{n-1} \cdot y^{n-1} k_{0}(y) \omega_{f}\left(x_{0} ; 4 \varepsilon y\right) \varepsilon d y=
\end{gathered}
$$

$$
\begin{aligned}
& =\int_{0}^{\infty} y^{n-1} \cdot k_{0}(y) \cdot \omega_{f}\left(x_{0} ; 4 \varepsilon y\right) d y= \\
& =\int_{0}^{\frac{1}{\sqrt{\varepsilon}}} y^{n-1} \cdot k_{0}(y) \cdot \omega_{f}\left(x_{0} ; 4 \varepsilon y\right) d y+ \\
& +\int_{\frac{1}{\sqrt{\varepsilon}} y^{n-1} \cdot k_{0}(y) \cdot \omega_{f}\left(x_{0} ; 4 \varepsilon y\right) d y \leq}^{\infty} \\
& \leq \omega_{f}\left(x_{0} ; 4 \sqrt{\varepsilon}\right) \cdot \int_{0}^{\infty} y^{n-1} k_{0}(y) d y+ \\
& \quad+\int_{\frac{1}{\sqrt{\varepsilon}}}^{\infty} y^{n-1} k_{0}(y) \omega_{f}\left(x_{0} ; 4 y\right) d y .
\end{aligned}
$$

If to consider, that

$$
\begin{gathered}
+\infty>\int_{R^{n}} k(x) d x=\int_{0}^{\infty} t^{n-1} \int_{S^{n-1}} k_{0}(|t \xi|) d \sigma_{\xi} d t= \\
=\left|S^{n-1}\right| \cdot \int_{0}^{\infty} k_{0}(t) t^{n-1} d t
\end{gathered}
$$

where $\left|S^{n-1}\right|$ is surface area of unit sphere $S^{n-1}, d \sigma_{\xi}$ is Lebesgue's measure on the sphere $S^{n-1}$, then from the inequalities (3.1), (3.6) and by condition (3.5) we receive the demanded statement.

Corollary 3.2 [16]. Let kernel $K(x)$ satisfies the conditions of Theorem 3.1 and $f \in L^{p}\left(R^{n}\right), 1 \leq p \leq \infty$, $x_{0} \in R^{n}$ is Lebesgue point of $f$. Then

$$
\lim _{\varepsilon \rightarrow 0} K_{\varepsilon} f\left(x_{0}\right)=l_{f}\left(x_{0}\right) \text {. }
$$

By theorem 3.1 the following statement for Poisson integral is obtained.

Corollary 3.3. Let $P(x)$ be Poisson kernel, $P_{\varepsilon}(x):=$ $\varepsilon^{-n} P\left(\frac{x}{\varepsilon}\right), \quad \varepsilon>0, \quad x \in R^{n}, \quad f \in L_{l o c}\left(R^{n}\right), \quad x_{0} \in R^{n} \quad$ is Lebesgue point of the function $f$ and

$$
\int_{1}^{\infty} \frac{\omega_{f}\left(x_{0} ; t\right)}{t^{2}} d t<+\infty
$$

Then the following inequality is satisfied:

$$
\left|\left(P_{\varepsilon} * f\right)\left(x_{0}\right)-l_{f}\left(x_{0}\right)\right| \leq c \cdot \varepsilon \int_{\varepsilon}^{\infty} \frac{\omega_{f}\left(x_{0} ; t\right)}{t^{2}} d t, \varepsilon>0,
$$

where $c>0$ is only depended on $n$, and hence $\lim _{\varepsilon \rightarrow+0}\left(P_{\varepsilon} * f\right)\left(x_{0}\right)=l_{f}\left(x_{0}\right)$.

It is easy to see that for the Poisson kernel $P(x)$ the conditions of Corollary 3.2 are satisfied. Therefore, if $f \in L^{p}\left(R^{n}\right), 1 \leq p \leq \infty$ and $x_{0}$ is Lebesgue point of the function $f$, we have the equality $\lim _{\varepsilon \rightarrow+0}\left(P_{\varepsilon} * f\right)\left(x_{0}\right)=$ 
$l_{f}\left(x_{0}\right)$.

Theorem 3.2. Let $x_{0} \in R^{n}$ and

1) $K(x)$ is nonnegative kernel such, that $K(x) \equiv$ $k_{0}(|x|), x \in R^{n}$, where $k_{0}(t)$ is monotone decreases on $[0,+\infty)$

2) $\omega(\delta)$ is positive monotone increasing function on $(0,+\infty)$ and satisfying $\lim _{\delta \rightarrow+0} \omega(\delta)=0$, for which there is a number $c_{0}>0$ such that $\omega(2 \delta) \leq c_{0} \cdot \omega(\delta)$, $\delta \in(0,+\infty)$.

Then there is a function $f_{0} \in L_{l o c}\left(R^{n}\right)$ such that, $\omega_{f_{0}}\left(x_{0} ; \delta\right)=\omega(\delta), \quad \delta \in(0,+\infty)^{1}$, and

$$
\begin{gathered}
\left|K_{\varepsilon} f_{0}\left(x_{0}\right)-s_{f_{0}}\left(x_{0}\right)\right| \geq \\
\geq c \cdot \varepsilon^{-n} \int_{0}^{\infty} t^{n-1} k_{0}\left(\frac{t}{\varepsilon}\right) \omega_{f_{0}}\left(x_{0} ; 4 t\right) d t, \quad \varepsilon>0 .
\end{gathered}
$$

Proof. Let $f_{0}(x)=\omega\left(\left|x-x_{0}\right|\right), x \in R^{n}$. Then

$$
\begin{gathered}
\lim _{r \rightarrow+0} \frac{1}{\left|B\left(x_{0}, r\right)\right|} \int_{B\left(x_{0}, r\right)} f_{0}(t) d t= \\
=\lim _{r \rightarrow+0} \frac{1}{\left|B\left(x_{0}, r\right)\right|} \int_{B\left(x_{0}, r\right)} \omega\left(\left|t-x_{0}\right|\right) d t=0,
\end{gathered}
$$

i.e. $s_{f_{0}}\left(x_{0}\right)=0$. Therefore

$$
\begin{gathered}
\omega_{f_{0}}\left(x_{0} ; \delta\right)=\sup _{0<r \leq \delta} \frac{1}{\left|B\left(x_{0}, r\right)\right|} \int_{B\left(x_{0}, r\right)}\left|f_{0}(t)-s_{f_{0}}\left(x_{0}\right)\right| d t= \\
=\sup _{0<r \leq \delta} \frac{1}{\left|B\left(x_{0}, r\right)\right|} \int_{B\left(x_{0}, r\right)} \omega\left(\left|t-x_{0}\right|\right) d t= \\
=\sup _{0<r \leq \delta} \frac{1}{|B(0, r)|} \int_{B(0, r)} \omega(|t|) d t .
\end{gathered}
$$

From here we get that, $\omega_{f_{0}}\left(x_{0} ; \delta\right) \leq \omega(\delta), \delta \in(0,+\infty)$. By (3.7) also obtained that,

$$
\begin{gathered}
\omega_{f_{0}}\left(x_{0} ; \delta\right) \geq \frac{1}{|B(0, \delta)|} \int_{B(0, \delta)} \omega(|t|) d t \geq \\
\geq \frac{1}{|B(0, \delta)|} \int_{B(0, \delta) \backslash B\left(0, \frac{\delta}{2}\right)} \omega(|t|) d t \geq \\
\geq \omega\left(\frac{\delta}{2}\right) \cdot \frac{1}{|B(0,1)| \cdot \delta^{n}} \cdot|B(0,1)| \cdot\left(\delta^{n}-\left(\left(\frac{\delta}{2}\right)\right)^{n}\right)= \\
=\omega\left(\frac{\delta}{2}\right) \cdot \frac{2^{n}-1}{2^{n}} \geq \\
\geq \frac{1}{c_{0}} \cdot \omega(\delta) \cdot \frac{2^{n}-1}{2^{n}}=c_{1} \cdot \omega(\delta), \quad \delta>0 .
\end{gathered}
$$

Further we have

\footnotetext{
${ }^{1}$ i.e. $\exists c_{1}, c_{2}>0 \quad \forall \delta \in(0,+\infty): c_{1} \cdot \omega_{f_{0}}\left(x_{0} ; \delta\right) \leq \omega(\delta) \leq c_{2} \cdot \omega_{f_{0}}\left(x_{0} ; \delta\right)$.
}

$$
\begin{gathered}
\left|K_{\varepsilon} f_{0}\left(x_{0}\right)-s_{f_{0}}\left(x_{0}\right)\right|=\varepsilon^{-n} \int_{R^{n}} k_{0}\left(\frac{\left|x_{0}-t\right|}{\varepsilon}\right) f_{0}(t) d t= \\
=\varepsilon^{-n} \int_{R^{n}} k_{0}\left(\frac{\left|x_{0}-t\right|}{\varepsilon}\right) \omega\left(\left|t-x_{0}\right|\right) d t= \\
=\varepsilon^{-n} \int_{R^{n}} k_{0}\left(\frac{|t|}{\varepsilon}\right) \omega(|t|) d t= \\
=\varepsilon^{-n} \int_{0}^{\infty} t^{n-1}\left(\int_{s^{n-1}} k_{0}\left(\frac{|t \xi|}{\varepsilon}\right) \omega(|t \xi|) d \sigma_{\xi}\right) d t= \\
=\left|S^{n-1}\right| \cdot \varepsilon^{-n} \int_{0}^{\infty} k_{0}\left(\frac{t}{\varepsilon}\right) \cdot t^{n-1} \omega(t) d t \geq \\
\geq \frac{1}{c_{0}^{2}} \cdot\left|S^{n-1}\right| \cdot \varepsilon^{-n} \int_{0}^{\infty} k_{0}\left(\frac{t}{\varepsilon}\right) \cdot t^{n-1} \omega(4 t) d t \geq \\
\geq \frac{1}{c_{0}^{2}} \cdot\left|S^{n-1}\right| \cdot \varepsilon^{-n} \int_{0}^{\infty} k_{0}\left(\frac{t}{\varepsilon}\right) \cdot t^{n-1} \omega_{f_{0}}\left(x_{0} ; 4 t\right) d t \\
\varepsilon>0 .
\end{gathered}
$$

This theorem shows unimprovability of estimations (3.1) in a certain class of kernels.

\section{Approximation in Terms of the Mean Oscillation}

Theorem 4.1. Let the kernel $K(x)$ satisfies conditions of the theorem B, $f \in L_{l o c}\left(R^{n}\right), x_{0}$ is Lebesgue point of $f$ and let satisfies the following conditions also:

1) $\int_{1}^{\infty} t^{n-1} k_{0}(t) m_{f}\left(x_{0} ; 4 t\right) d t<+\infty$,

2) $\int_{0}^{1}\left(\frac{1}{t} \int_{0}^{t} x^{n-1} k_{0}(x) d x\right) m_{f}\left(x_{0} ; 4 t\right) d t<+\infty$,

3) $\int_{1}^{\infty}\left(\frac{1}{t} \int_{t}^{\infty} x^{n-1} k_{0}(x) d x\right) m_{f}\left(x_{0} ; 4 t\right) d t<+\infty$.

Then $\lim _{\varepsilon \rightarrow+0} K_{\varepsilon} f\left(x_{0}\right)=l_{f}\left(x_{0}\right)$.

Proof. Since $x_{0} \in R^{n}$ is Lebesgue point of function $f$, then by the Theorem A the equality $\lim _{\delta \rightarrow+0} m_{f}\left(x_{0} ; \delta\right)=0$ is satisfied and the finite limit $s_{f}\left(x_{0}\right):=\lim _{\varepsilon \rightarrow+0} f_{B\left(x_{0}, \varepsilon\right)}=$ $l_{f}\left(x_{0}\right)$ exists.

Let's show that, if the conditions 1), 2) and 3) are satisfied, then all terms on the right hand side of inequality (2.1) approaches to zero as $\varepsilon \rightarrow+0$. We can assume that $0<\varepsilon \leq 1$. Then we get

$$
\int_{0}^{\infty} t^{n-1} k_{0}(t) m_{f}\left(x_{0} ; 4 \varepsilon t\right) d t=
$$




$$
\begin{aligned}
& \frac{1}{\sqrt{\varepsilon}} \\
& =\int_{0} t^{n-1} k_{0}(t) m_{f}\left(x_{0} ; 4 \varepsilon t\right) d t+ \\
& +\int_{\frac{1}{\sqrt{\varepsilon}}}^{\infty} t^{n-1} k_{0}(t) m_{f}\left(x_{0} ; 4 \varepsilon t\right) d t \leq \\
& \leq m_{f}\left(x_{0} ; 4 \sqrt{\varepsilon}\right) \int_{0}^{\infty} t^{n-1} k_{0}(t) d t+ \\
& +\int_{\frac{1}{\sqrt{\varepsilon}}}^{\infty} t^{n-1} k_{0}(t) m_{f}\left(x_{0} ; 4 t\right) d t \\
& \int_{0}^{\varepsilon} \frac{m_{f}\left(x_{0} ; t\right)}{t}\left(\int_{0}^{\frac{t}{4 \varepsilon}} x^{n-1} k_{0}(x) d x\right) d t= \\
& =\int_{0}^{\frac{1}{4}} \frac{m_{f}\left(x_{0} ; 4 \varepsilon y\right)}{4 \varepsilon y}\left(\int_{0}^{y} x^{n-1} k_{0}(x) d x\right) 4 \varepsilon d y= \\
& =\int_{0}^{\frac{1}{4}}\left(\frac{1}{y} \int_{0}^{y} x^{n-1} k_{0}(x) d x\right) m_{f}\left(x_{0} ; 4 \varepsilon y\right) d y \leq \\
& \leq \int_{0}^{m_{f}\left(x_{0} ; \varepsilon\right)}\left(\frac{1}{y} \int_{0}^{y} x^{n-1} k_{0}(x) d x\right) m_{f}\left(x_{0} ; 4 y\right) d y+ \\
& +m_{f}\left(x_{0} ; \varepsilon\right) \int_{m_{f}\left(x_{0} ; \varepsilon\right)}^{\frac{1}{4}}\left(\frac{1}{y} \int_{0}^{y} x^{n-1} k_{0}(x) d x\right) d y
\end{aligned}
$$

where $\varepsilon$ is positive number such that $0<\varepsilon \leq 1$ and $m_{f}\left(x_{0} ; \varepsilon\right) \leq \frac{1}{4}$;

$$
\begin{aligned}
& \int_{\varepsilon}^{\infty} \frac{m_{f}\left(x_{0} ; t\right)}{t}\left(\int_{\frac{t}{4 \varepsilon}}^{\infty} x^{n-1} k_{0}(x) d x\right) d t= \\
= & \int_{\frac{1}{4}}^{\infty} \frac{m_{f}\left(x_{0} ; 4 \varepsilon y\right)}{4 \varepsilon y}\left(\int_{y}^{\infty} x^{n-1} k_{0}(x) d x\right) 4 \varepsilon d y= \\
= & \int_{\frac{1}{4}}^{\infty}\left(\frac{1}{y} \int_{y}^{\infty} x^{n-1} k_{0}(x) d x\right) m_{f}\left(x_{0} ; 4 \varepsilon y\right) d y \leq \\
\leq & \left\{\int_{\frac{1}{4}}^{\frac{1}{\sqrt{\varepsilon}}}\left(\frac{1}{y} \int_{y}^{\infty} x^{n-1} k_{0}(x) d x\right) d y\right\} \cdot m_{f}\left(x_{0} ; 4 \sqrt{\varepsilon}\right)+
\end{aligned}
$$

$$
\begin{gathered}
+\int_{\frac{1}{\sqrt{\varepsilon}}}^{\infty}\left(\frac{1}{y} \int_{y}^{\infty} x^{n-1} k_{0}(x) d x\right) m_{f}\left(x_{0} ; 4 y\right) d y \leq \\
\leq \frac{m_{f}\left(x_{0} ; 4 \sqrt{\varepsilon}\right)}{m_{f}\left(x_{0} ; 1\right)} \int_{\frac{1}{4}}^{\infty}\left(\frac{1}{y} \int_{y}^{\infty} x^{n-1} k_{0}(x) d x\right) m_{f}\left(x_{0} ; 4 y\right) d y+ \\
+\int_{\frac{1}{\sqrt{\varepsilon}}}^{\infty}\left(\frac{1}{y} \int_{y}^{\infty} x^{n-1} k_{0}(x) d x\right) m_{f}\left(x_{0} ; 4 y\right) d y
\end{gathered}
$$

By considering that in the case of satisfying conditions of the theorem the following integral

$$
\int_{0}^{\infty} x^{n-1} k_{0}(x) d x
$$

converges, from inequality (4.1), (4.2) and (4.3) the required assertion is obtained.

Corollary 4.1. Let the kernel $K(x)$ satisfies conditions of the theorem $\mathrm{B}, f \in B M O, x_{0}$ is Lebesgue point of function $f$, the condition 2 ) is satisfied and

$$
\int_{1}^{\infty}\left(\frac{1}{t} \int_{t}^{\infty} x^{n-1} k_{0}(x) d x\right) d t<+\infty .
$$

Then $\lim _{\varepsilon \rightarrow+0} K_{\varepsilon} f\left(x_{0}\right)=l_{f}\left(x_{0}\right)$.

Corollary 4.2. Let $f \in L_{l o c}\left(R^{n}\right), x_{0}$ is Lebesgue point of function $f$ and let the following condition is satisfied:

$$
\begin{gathered}
\exists \gamma>0 \sup \left\{(1+|t|)^{n+\gamma}|K(t)|: t \in R^{n}\right\}<+\infty, \\
\int_{1}^{\infty} t^{-1-\gamma} \cdot m_{f}\left(x_{0} ; t\right) d t<+\infty .
\end{gathered}
$$

Then $\lim _{\varepsilon \rightarrow+0} K_{\varepsilon} f\left(x_{0}\right)=l_{f}\left(x_{0}\right)$.

Proof. By the condition (4.4) follows that,

$$
\exists C>0 \quad \forall t \geq 0: \quad k_{0}(t) \leq C \cdot \frac{1}{(1+t)^{n+\gamma}} .
$$

Now let's show that conditions of theorem 4.1 are satisfied. We have that

$$
\begin{gathered}
\int_{1}^{\infty} t^{n-1} k_{0}(t) m_{f}\left(x_{0} ; 4 t\right) d t \leq \\
\leq C \cdot \int_{1}^{\infty} t^{n-1} \cdot \frac{1}{(1+t)^{n+\gamma}} \cdot m_{f}\left(x_{0} ; 4 t\right) d t \leq \\
\leq C_{1} \cdot \int_{1}^{\infty} \frac{m_{f}\left(x_{0} ; t\right)}{t^{1+\gamma}} d t<+\infty, \quad C_{1}=\text { const; } \\
\int_{0}^{1}\left(\frac{1}{t} \int_{0}^{t} x^{n-1} k_{0}(x) d x\right) m_{f}\left(x_{0} ; 4 t\right) d t \leq
\end{gathered}
$$




$$
\begin{aligned}
& \leq C \cdot \int_{0}^{1}\left(\frac{1}{t} \int_{0}^{t} \frac{x^{n-1} d x}{(1+x)^{n+\gamma}}\right) m_{f}\left(x_{0} ; 4 t\right) d t \leq \\
& \leq C \cdot \int_{0}^{1}\left(\frac{1}{t} \int_{0}^{t} x^{n-1} d x\right) m_{f}\left(x_{0} ; 4 t\right) d t= \\
& =\frac{C}{n} \cdot \int_{0}^{1} t^{n-1} \cdot m_{f}\left(x_{0} ; 4 t\right) d t<+\infty ; \\
& \int_{1}^{\infty}\left(\frac{1}{t} \int_{t}^{\infty} x^{n-1} k_{0}(x) d x\right) m_{f}\left(x_{0} ; 4 t\right) d t \leq \\
& \leq C \cdot \int_{1}^{\infty}\left(\frac{1}{t} \int_{t}^{\infty} \frac{x^{n-1}}{x^{n+\gamma}} d x\right) m_{f}\left(x_{0} ; 4 t\right) d t= \\
& =C \cdot \int_{1}^{\infty}\left(\frac{1}{t} \int_{t}^{\infty} x^{-\gamma-1} d x\right) m_{f}\left(x_{0} ; 4 t\right) d t \leq \\
& \leq C_{2} \cdot \int_{1}^{\infty} \frac{m_{f}\left(x_{0} ; t\right)}{t^{1+\gamma}} d t<+\infty, \quad C_{2}=\text { const. }
\end{aligned}
$$

Thus, all conditions of the theorem 4.1 are satisfied and because of this $\lim _{\varepsilon \rightarrow+0} K_{\varepsilon} f\left(x_{0}\right)=l_{f}\left(x_{0}\right)$.

Note that, particularly, any function $f \in B M O$ satisfy condition (4.5) for all points $x_{0} \in R^{n}$. The kernel $K(x)$, which satisfies conditions (4.4) is called kernel of Fejer type.

Theorem 4.2. Let kernel $K(x)$ satisfies conditions of theorem B and $f \in L_{l o c}\left(R^{n}\right)$. Then for a finite value of right hand side we have the following inequality

$$
\begin{gathered}
\left\|f-K_{\varepsilon} f\right\|_{B M O} \leq \\
\leq C \cdot\left(M_{f}(\varepsilon)+\int_{0}^{\infty} x^{n-1} k_{0}(x) M_{f}(4 \varepsilon x) d x+\right. \\
+\int_{0}^{\varepsilon} \frac{M_{f}(t)}{t}\left(\int_{0}^{\frac{t}{4 \varepsilon}} x^{n-1} k_{0}(x) d x\right) d t+ \\
\left.+\int_{\varepsilon}^{\infty} \frac{M_{f}(t)}{t}\left(\int_{\frac{t}{4 \varepsilon}}^{\infty} x^{n-1} k_{0}(x) d x\right) d t\right), \quad \varepsilon>0,
\end{gathered}
$$

where the constant $C>0$ depends only on $n$ and $k_{0}$.

Proof. From inequality (2.1) we have

$$
\begin{gathered}
\left\|K_{\varepsilon} f-f_{B(\cdot ; \varepsilon)}\right\|_{B M O} \leq 2\left\|K_{\varepsilon} f-f_{B(\cdot ; \varepsilon)}\right\|_{L^{\infty}\left(R^{n}\right)} \leq \\
\quad \leq C\left(M_{f}(\varepsilon)+\int_{0}^{\infty} x^{n-1} k_{0}(x) M_{f}(4 \varepsilon x) d x+\right.
\end{gathered}
$$

$$
\begin{gathered}
+\int_{0}^{\varepsilon} \frac{M_{f}(t)}{t}\left(\int_{0}^{\frac{t}{4 \varepsilon}} x^{n-1} k_{0}(x) d x\right) d t+ \\
\left.+\int_{\varepsilon}^{\infty} \frac{M_{f}(t)}{t}\left(\int_{\frac{t}{4 \varepsilon}}^{\infty} x^{n-1} k_{0}(x) d x\right) d t\right), \quad \varepsilon>0 .
\end{gathered}
$$

It is known that (see [6])

$$
\left\|f-f_{B(\cdot ; \varepsilon)}\right\|_{B M O} \leq C \cdot M_{f}(\varepsilon), \quad \varepsilon>0,
$$

where $C>0$ is independent of $f$ and $\varepsilon$. From inequalities (4.7) and (4.8) the inequality (4.6) turns out.

Corollary 4.3. Let kernel $K(x)$ satisfies conditions of theorem $\mathrm{B}, f \in V M O$ and also satisfies the following conditions:

$\left.1^{0}\right) \int_{0}^{1}\left(\frac{1}{t} \int_{0}^{t} x^{n-1} k_{0}(x) d x\right) M_{f}(4 t) d t<+\infty$,

$\left.2^{0}\right) \int_{1}^{\infty}\left(\frac{1}{t} \int_{t}^{\infty} x^{n-1} k_{0}(x) d x\right) d t<+\infty$.

Then $\lim _{\varepsilon \rightarrow+0}\left\|K_{\varepsilon} f-f\right\|_{B M O}=0$.

Proof. It is enough to check up that under our assumptions all terms on the right hand side of the inequality (4.6) approaches to zero, with $\varepsilon \rightarrow+0$. If $f \in V M O$, then $\lim _{\varepsilon \rightarrow+0} M_{f}(\varepsilon)=0$. In addition, if $0<\varepsilon \leq 1$, then

$$
\begin{gathered}
\int_{0}^{\infty} x^{n-1} k_{0}(x) M_{f}(4 \varepsilon x) d x \leq M_{f}(4 \sqrt{\varepsilon}) \int_{0}^{\infty} x^{n-1} k_{0}(x) d x+ \\
+\|f\|_{B M O} \cdot \int_{\frac{1}{\sqrt{\varepsilon}}}^{\infty} x^{n-1} k_{0}(x) d x \\
\int_{0}^{\varepsilon} \frac{M_{f}(t)}{t}\left(\int_{0}^{\frac{t}{4 \varepsilon}} x^{n-1} k_{0}(x) d x\right) d t= \\
=\int_{0}^{\frac{1}{4}}\left(\frac{1}{y} \int_{0}^{y} x^{n-1} k_{0}(x) d x\right) M_{f}(4 \varepsilon y) d y \leq \\
\leq \int_{0}^{M_{f}(\varepsilon)}\left(\frac{1}{y} \int_{0}^{y} x^{n-1} k_{0}(x) d x\right) M_{f}(4 y) d y+ \\
+M_{f}(\varepsilon) \int_{M_{f}(\varepsilon)}^{\frac{1}{4}}\left(\frac{1}{y} \int_{0}^{y} x^{n-1} k_{0}(x) d x\right) d y
\end{gathered}
$$

where $\varepsilon$ is positive number such that $\varepsilon \leq 1$ and $M_{f}(\varepsilon) \leq$ $\frac{1}{4}$; 


$$
\begin{gathered}
\int_{\varepsilon}^{\infty} \frac{M_{f}(t)}{t}\left(\int_{\frac{t}{4 \varepsilon}}^{\infty} x^{n-1} k_{0}(x) d x\right) d t= \\
=\int_{\frac{1}{4}}^{\infty}\left(\frac{1}{y} \int_{y}^{\infty} x^{n-1} k_{0}(x) d x\right) M_{f}(4 \varepsilon y) d y \leq \\
\leq\left(\int_{\frac{1}{4}}^{\frac{1}{\sqrt{\varepsilon}}}\left(\frac{1}{y} \int_{y}^{\infty} x^{n-1} k_{0}(x) d x\right) d y\right) \cdot M_{f}(4 \sqrt{\varepsilon})+ \\
+\int_{\frac{1}{\sqrt{\varepsilon}}}^{\infty}\left(\frac{1}{y} \int_{y}^{\infty} x^{n-1} k_{0}(x) d x\right) M_{f}(4 y) d y \leq \\
\frac{1}{M_{f}(1)} \cdot M_{f}(4 \sqrt{\varepsilon}) \cdot \int_{\frac{1}{4}}^{\infty}\left(\frac{1}{y} \int_{y}^{\infty} x^{n-1} k_{0}(x) d x\right) M_{f}(4 y) d y+ \\
+\int_{\frac{1}{\sqrt{\varepsilon}}}^{\infty}\left(\frac{1}{y} \int_{y}^{\infty} x^{n-1} k_{0}(x) d x\right) M_{f}(4 y) d y .
\end{gathered}
$$

It is easy to check that if conditions $1^{0}$ ) and $2^{0}$ ) are satisfied, then the integral $\int_{0}^{\infty} x^{n-1} k_{0}(x) d x$ converges. Therefore the obtained relations show the validity of the required statement by virtue of inequality (4.6).

Note that, if $K(x)$ is Poisson kernel and $f \in B M O$, then the conditions $1^{0}$ ) and $2^{0}$ ) are satisfied. Thus, if $f \in V M O$ and $K$ is Poisson kernel, then $\lim _{\varepsilon \rightarrow+0}\left\|K_{\varepsilon} f-f\right\|_{B M O}=0$. It is known that, if $f \in B M O(R), K$ is Poisson kernel (for $n=1$ ) and $\lim _{\varepsilon \rightarrow+0}\left\|K_{\varepsilon} f-f\right\|_{B M O}=0$, then $f \in V M O$ (see [4]).

Corollary 4.4. Let kernel $K(x)$ satisfies condition (4.4), $f \in L_{l o c}\left(R^{n}\right)$ and

$$
\int_{1}^{\infty} \frac{M_{f}(t)}{t^{1+\gamma}} d t<+\infty
$$

Then the following inequality is true

$$
\begin{aligned}
& \left\|f-K_{\varepsilon} f\right\|_{B M O} \leq \\
& \leq C \cdot \varepsilon^{\gamma} \int_{\varepsilon}^{\infty} \frac{M_{f}(t)}{t^{1+\gamma}} d t, \varepsilon>0,
\end{aligned}
$$

where the constant $C>0$ is independent of $f$ and $\varepsilon$.

Proof. Estimate terms on the right hand side of inequality (4.6). We have

$$
\int_{0}^{\infty} x^{n-1} k_{0}(x) M_{f}(4 \varepsilon x) d x=
$$

$$
\begin{aligned}
& =\int_{0}^{\frac{1}{4}} x^{n-1} k_{0}(x) M_{f}(4 \varepsilon x) d x+\int_{\frac{1}{4}}^{\infty} x^{n-1} k_{0}(x) M_{f}(4 \varepsilon x) d x \leq \\
& \leq \mathrm{c}_{1} \cdot \int_{0}^{\frac{1}{4}} x^{n-1} \cdot M_{f}(4 \varepsilon x) d x+ \\
& +\mathrm{c}_{2} \cdot \int_{\frac{1}{4}}^{\infty} x^{n-1} \cdot \frac{1}{x^{n+\gamma}} \cdot M_{f}(4 \varepsilon x) d x \leq \\
& \leq c_{3} \cdot\left(M_{f}(\varepsilon)+\varepsilon^{\gamma} \int_{\varepsilon}^{\infty} \frac{M_{f}(x)}{x^{1+\gamma}} d x\right) \leq c_{4} \cdot \varepsilon^{\gamma} \int_{\varepsilon}^{\infty} \frac{M_{f}(t)}{t^{1+\gamma}} d t ; \\
& \int_{0}^{\varepsilon} \frac{M_{f}(t)}{t}\left(\int_{0}^{\frac{t}{4 \varepsilon}} x^{n-1} k_{0}(x) d x\right) d t \leq c_{5} \cdot \int_{0}^{\varepsilon} \frac{M_{f}(t)}{t}\left(\frac{t}{\varepsilon}\right)^{n} d t= \\
& =c_{5} \cdot \frac{1}{\varepsilon^{n}} \int_{0}^{\varepsilon} M_{f}(t) t^{n-1} d t \leq \\
& \leq c_{6} \cdot M_{f}(\varepsilon) \leq c_{7} \cdot \varepsilon^{\gamma} \int_{\varepsilon}^{\infty} \frac{M_{f}(t)}{t^{1+\gamma}} d t ; \\
& \int_{\varepsilon}^{\infty} \frac{M_{f}(t)}{t}\left(\int_{\frac{t}{4 \varepsilon}}^{\infty} x^{n-1} k_{0}(x) d x\right) d t \leq \\
& \leq c_{8} \cdot \int_{\varepsilon}^{\infty} \frac{M_{f}(t)}{t}\left(\int_{\frac{t}{4 \varepsilon}}^{\infty} x^{n-1} \cdot \frac{1}{x^{n+\gamma}} d x\right) d t \leq \\
& \leq c_{9} \cdot \varepsilon^{\gamma} \int_{\varepsilon}^{\infty} \frac{M_{f}(t)}{t^{1+\gamma}} d t
\end{aligned}
$$

where $c_{i}(i=1,2, \ldots, 9)$ are positive constants, not depending on $f$ and $\varepsilon$. By obtained inequalities from inequality (4.6) we get estimation (4.10).

Corollary 4.5. Let kernel $K(x)$ satisfies condition (4.4), $f \in B M O_{\varphi}$,

$$
\varepsilon^{\gamma} \int_{\varepsilon}^{\infty} \frac{\varphi(t)}{t^{1+\gamma}} d t=O(\varphi(\varepsilon)), \quad \varepsilon>0 .
$$

Then the following relation is true

$$
\left\|f-K_{\varepsilon} f\right\|_{B M O}=O(\varphi(\varepsilon)), \quad \varepsilon>0 .
$$




\section{References}

[1] Butzer P.L., Nessel R.J. Fourier analysis and approximation. Vol.1: One-Dimensional Theory. New York and London, 1971.

[2] Calderon A.P., Zygmund A. On the existence of certain singular integrals. Acta. Math., 1952, v.88, pp.85-139.

[3] Gadzhiev N.M., Rzaev R.M. On the order of locally summable functions approximation by singular integrals. Funct. Approx. Comment. Math., 1992, v.20, pp.35-40.

[4] Garnett J.B. Bounded analytic functions. Academic Press Inc., New York, 1981.

[5] Golubov B.I. On asymptotics of multiple singular integrals for differentiable functions. Matem. Zametki, 1981, v.30, No5, pp.749-762 (Russian).

[6] Janson S. On functions with conditions on the mean oscillation. Ark. math., 1976, v.14, No2, pp. 189-196.

[7] John F., Nirenberg L. On functions of bounded mean oscillation. Comm. Pure Appl. Math., 1961, v.14, pp.415-426.

[8] Kerman R.A. Pointwise convergent approximate identities of dilated radially decreasing kernels. Proc. Amer. Math. Soc., 1987, v.101, No1, pp.41-44.

[9] Rzaev R.M. On approximation of essentially continuous functions by singular integrals. Izv. Vuzov. Matematika, 1989, No3, pp.57-62 (Russian).

[10] Rzaev R.M. On approximation of locally summable functions by singular integrals in terms of mean oscillation and some applications. Preprint Inst. Phys. Natl. Acad. Sci. Azerb., 1992, №1, p.1-43 (Russian).

[11] Rzaev R.M. A multidimensional singular integral operator in the spaces defined by conditions on the $k$-th order mean oscillation. Dokady Mathematics, 1997, v.56, No2, pp.747-749.

[12] Rzaev R.M., Aliyeva L.R. On local properties of functions and singular integrals in terms of the mean oscillation. Cent. Eur. J. Math., 2008, v.6, No4, p.595-609.

[13] Sarason D. Functions of vanishing mean oscillation. Trans. Amer. Math. Soc., 1975, v.207, pp. 391-405.

[14] Spanne S. Some function spaces defined using the mean oscillation over cubes. Ann. Scuola Norm. Sup. Pisa, 1965, v.19, No4, pp.593-608.

[15] Stein E.M., Singular integrals and differentiability properties of functions. Princeton University Press. Princeton, New J., 1970.

[16] Stein E.M., Weiss G. Introduction to Fourier Analysis on Euclidean spaces. Princeton University Press. Princeton, New J., 1971. 